\title{
A prognostic model for lung adenocarcinoma patient survival with a focus on four miRNAs
}

\author{
XIANQIU LI, ZHAOLING AN, PEIHUI LI and HAIHUA LIU \\ Department of Pharmacy, Weifang People's Hospital,Weifang, Shandong 261041, P.R. China
}

Received March 6, 2017; Accepted June 15, 2017

DOI: $10.3892 / \mathrm{ol} .2017 .6481$

\begin{abstract}
There is currently no effective biomarker for determining the survival of patients with lung adenocarcinoma. The purpose of the present study was to construct a prognostic survival model using microRNA (miRNA) expression data from patients with lung adenocarcinoma. miRNA data were obtained from The Cancer Genome Atlas, and patients with lung adenocarcinoma were divided into either the training or validation set based on the random allocation principle. The prognostic model focusing on miRNA was constructed, and patients were divided into high-risk or low-risk groups as per the scores, to assess their survival time. The 5-year survival rate from the subgroups within the high- and lowrisk groups was assessed. P-values of the prognostic model in the total population, the training set and validation set were $0.0017,0.01986$ and 0.02773 , respectively, indicating that the survival time of the lung adenocarcinoma high-risk group was less than that of the low-risk group. Thus, the model had a good assessment effectiveness for the untreated group $(\mathrm{P}=0.00088)$ and the Caucasian patient group $(\mathrm{P}=0.00043)$. In addition, the model had the best prediction effect for the 5-year survival rate of the Caucasian patient group (AUC=0.629). In conclusion, the prognostic model developed in the present study can be used as an independent prognostic model for patients with lung adenocarcinoma.
\end{abstract}

\section{Introduction}

Lung adenocarcinoma is the most common form of lung cancer and it belongs to the histologic subgroup of non-small cell lung cancer $(1,2)$. This cancer type grows slowly but can undergo hematogenous metastasis at an early stage. The prognosis for survival is poorer for lung adenocarcinoma than squamous carcinoma, and its 5-year survival rate after surgical removal is less than 10\% (3-6). In the United States, almost $40 \%$ of lung cancers are adenocarcinoma and usually

Correspondence to: Dr Haihua Liu, Department of Pharmacy, Weifang People's Hospital, 151 Guangwen Street, Kuiwen, Weifang, Shandong 261041, P.R. China

E-mail: h5u7e9@163.com

Key words: lung adenocarcinoma, prognostic model, microRNAs originate from the surrounding lung tissue. The incidence of lung adenocarcinoma varies with age, and is more common among female subjects. The number of newly diagnosed cases has been on the increase in many western developed countries in recent decades, and it now constitutes the most common type of lung cancer among smokers, replacing squamous cell lung cancer (7-9).

A microRNA (miRNA) is a short single highly-conserved non-coding RNA that is important in the expression and functional regulation of eukaryotic genomes (e.g., proliferation, apoptosis, migration and angiogenesis) and these biological processes are integral for tumor formation and development (10-12). miR-378 (11) inhibits migration and invasion of prostate cancer cell, and promotes apoptosis. In addition, Zhou et al (13) found that miR-590-5p inhibited breast cancer cells, thereby providing a novel therapeutic approach for breast cancer patients. The Cancer Genome Atlas (TCGA), is an existing relatively authoritative sequencing database, that contains a variety of common tumors (14). The purpose of this database is to better understand the molecular basis of cancer through the application of genome analysis technologies, identifying mutations in DNA sequence, copy number variation and alterations in methylation status.

The aim of the present study was to combine partial genomic data to determine the prognosis of patients with lung adenocarcinoma.

\section{Materials and methods}

miRNA and patient data. Level 3 data of miRNA expression profile and the corresponding clinical data were downloaded from the TCGA (14). All the data were publically available. The downloaded miRNA expression data and clinical data were integrated, and patients with lung adenocarcinoma were selected for inclusion. Patients with lung adenocarcinoma whose age was unknown and those whose survival time was $<30$ days were excluded.

The downloaded data from TCGA included tissue miRNA data from 521 patients with lung adenocarcinoma and miRNA expression data of 46 cases of para-carcinoma tissue. To screen differentially expressed miRNA, the selection criteria were set to be log fold change $>1$ and $\mathrm{P}<0.05$. The up- and downregulated expression of miRNA in lung adenocarcinoma tissue was screened, and standardized treatment was conducted on the miRNA expression. In addition, the thermograph of tissue 


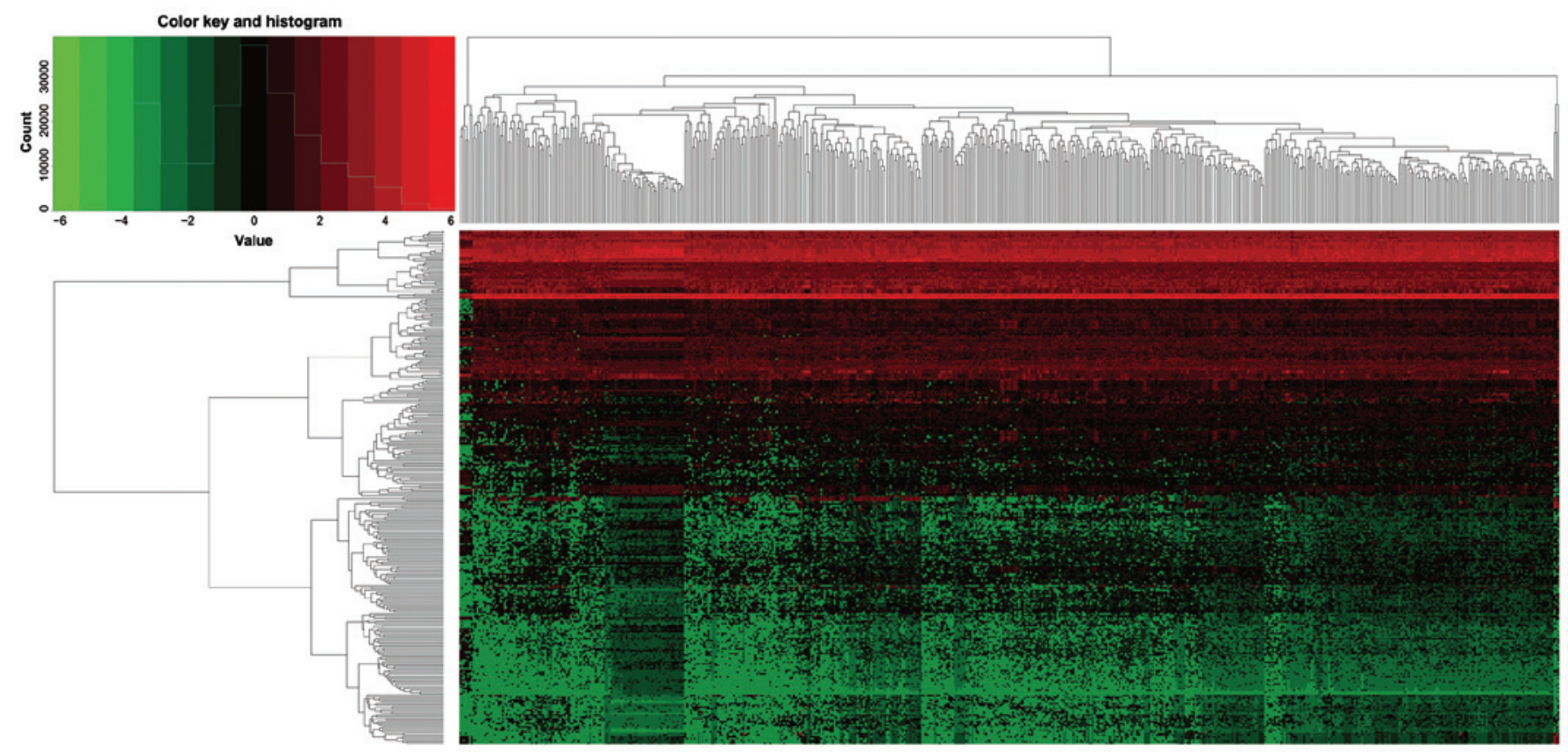

Figure 1. Heat map for microarray analysis of a total of 521 patients with lung adenocarcinoma tissue and 46 cases of para-carcinoma tissue. Red, high expression; green, low expression.

miRNA expression of patients with lung adenocarcinoma was drawn through pheatmap R of software R (15).

Establishment of prognostic model. To construct the prognosis model focusing on miRNA for lung adenocarcinoma, a log2 transform was conducted on the expression level of miRNA. A total of 478 cases with lung adenocarcinoma were randomly divided into either the training or test set, and the Chi-square test was applied to test whether there was statistical significance at each stage from the two sets $(\mathrm{P}<0.05)$. The miRNA that was closely associated with survival time of the patients in the training set were selected through univariate Cox proportional hazards regression $(\mathrm{P}<0.001)$. Next, miRNA was constructed into the prognostic model with the multivariate Cox regression. The optimal prognostic model was selected based on the Akaike information criterion (16). The median value of the model of the training set was regarded as the cut-off value, and the patients with lung adenocarcinoma in the training set were divided into either the high-risk or low-risk group. A Kaplan-Meier (KM) survival plot was constructed and if $\mathrm{P}<0.05$ (the model was established in the training set) the model was entered into the verification set. The median value of the training set model was regarded as the cut-off value. In addition, the patients with lung adenocarcinoma in the verification set were divided into high- or low-risk groups. KM survival curve was constructed and if $\mathrm{P}<0.05$, it showed that the prognosis model was established. After the model was established, KM survival curves were respectively drawn for various subgroups (e.g., male vs. female, age $>65$ or $\leq 65$ years, type of treatment). Then, receiver operating characteristic (ROC) curve of 5-year survival rate of subgroups was drawn using the ROC package of software R (17).

Cox regression. A single factor Cox regression was used to evaluate the relationship between clinical characteristics and survival time of patients with lung adenocarcinoma. The clinical features that were closely related to the survival were screened, and they, together with prognosis model were analyzed using multivariate Cox regression model to explore whether the prognosis model could be used as an independent predictor of patient's prognosis with lung adenocarcinoma.

\section{Results}

A total of 1,881 miRNA expression spectrum (level 3) data of 521 patients with lung adenocarcinoma tissue and 46 cases of para-carcinoma tissue were downloaded from TCGA. A total of 309 differentially expressed miRNAs were screened, including 188 upregulated miRNAs and 121 downregulated miRNAs (Fig. 1). hsa-miR-210, hsa-miR-708 and hsa-miR-96 were the three most significantly upregulated miRNAs, while hsa-miR-486-1, hsa-miR-486-2 and hsa-miR-4732 were the three most significantly downregulated miRNAs. According to the inclusion criteria, the data from 478 patients with lung adenocarcinoma were analyzed. These patients were sub-divided into the training $(\mathrm{n}=239)$ or test set $(\mathrm{n}=239)$, based on the principle of random distribution (Table I). There was no statistical difference between the stages from the training or validation set $(\mathrm{P}>0.05)$.

For the prognosis model constructed in the present study, the prognostic score $=44.488 \times$ (expression quantity of hsa-miR-101-1) - 1.673 x (expression quantity of hsa-miR-200a) $+0.428 \times$ (expression quantity of hsa-miR-4661) $+0.515 x$ (expression quantity of hsa-miR-450a-2). Of the four miRNAs, hsa-miR-4661 and hsa-miR-4661 had an upregulated expression, while hsa-miR-101-1 and hsa-miR-200a had a downregulated expression. The significance (P-value) from the KM survival curve of the prognostic model in the training set, the validation set, and the total number of patients, was 0.01986 (Fig. 2), 0.02773 (Fig. 3) and 0.0017 (Fig. 4), respectively, 
Table I. Patient demographics and tumor characteristics from the training and validation set.

\begin{tabular}{|c|c|c|c|c|}
\hline Covariates & Total $(n=478)$ & Training set $(n=239)$ & Testing set $(n=239)$ & P-value \\
\hline Age (years) & & & & 0.833 \\
\hline$\leq 65$ & 232 & 121 & 111 & \\
\hline$>65$ & 246 & 118 & 128 & \\
\hline Pathological stages & & & & 0.90 \\
\hline I & 259 & 134 & 125 & \\
\hline II & 111 & 53 & 58 & \\
\hline III & 78 & 33 & 45 & \\
\hline IV & 24 & 17 & 7 & \\
\hline NA & 6 & 2 & 4 & \\
\hline Pathology T stages & & & & 0.87 \\
\hline $\mathrm{T} 1$ & 163 & 85 & 78 & \\
\hline $\mathrm{T} 2$ & 250 & 121 & 129 & \\
\hline $\mathrm{T} 3$ & 44 & 22 & 22 & \\
\hline $\mathrm{T} 4$ & 18 & 9 & 9 & \\
\hline $\mathrm{Tx}$ & 3 & 2 & 1 & \\
\hline Pathology N stages & & & & 0.83 \\
\hline No & 309 & 161 & 148 & \\
\hline N1 & 87 & 40 & 47 & \\
\hline N2-N3 & 70 & 32 & 38 & \\
\hline NX-NA & 12 & 6 & 6 & \\
\hline Pathology M stages & & & & 0.91 \\
\hline M0 & 315 & 155 & 160 & \\
\hline M1 & 23 & 17 & 6 & \\
\hline MX & 136 & 65 & 71 & \\
\hline NA & 4 & 2 & 2 & \\
\hline Sex & & & & 0.86 \\
\hline Male & 222 & 113 & 109 & \\
\hline Female & 256 & 126 & 130 & \\
\hline Radiation therapy & & & & 0.75 \\
\hline No & 374 & 192 & 182 & \\
\hline Yes & 59 & 24 & 35 & \\
\hline NA & 45 & 23 & 22 & \\
\hline Ethnicity & & & & 0.86 \\
\hline Asian & 7 & 4 & 3 & \\
\hline Caucasian & 373 & 191 & 182 & \\
\hline African or African-American & 52 & 23 & 29 & \\
\hline NA & 46 & 21 & 25 & \\
\hline Status & & & & 0.79 \\
\hline Dead & 169 & 83 & 86 & \\
\hline Surviving & 309 & 156 & 153 & \\
\hline
\end{tabular}

NA, not applicable.

showing that the high-risk group of lung adenocarcinoma patients in the model had a shorter survival time than low-risk group. In addition, the model assessed each subgroup. The statistical significance (P-values) from the KM survival curve of the male, female, treatment, non-treatment, Caucasian group and non-Caucasian groups was 0.04055, 0.01487, $0.65091,0.00088,0.00043$ and 0.60825 , respectively
(Figs. 2-4). In the present study, only the ROC curve of the 5-year survival rate of the subgroups which KM survival curve $\mathrm{P}<0.05$ was utilized. The results showed that the prognosis model had the best diagnostic performance in the Caucasian group, with the area under the curve AUC $=0.629$; AUC of male group, female group and non-treatment group was 0.595 , 0.592 and 0.579 respectively. 
Table II. Association of clinical factors and the miRNA signature score with survival time from lung adenocarcinoma patients.

\begin{tabular}{|c|c|c|c|c|}
\hline \multirow[b]{2}{*}{ Variables } & \multicolumn{2}{|c|}{ Univariate analysis } & \multicolumn{2}{|c|}{ Multivariate analysis } \\
\hline & $\mathrm{HR}(95 \% \mathrm{CI})$ & P-value & HR $(95 \% \mathrm{CI})$ & P-value \\
\hline Age ( $>65$ vs. $\leq 65)$ & 1.18 & 0.307 & 1.31 & 0.03 \\
\hline Pathologic stages (IV vs. III vs. II vs. I) & 1.56 & $2.22 \mathrm{E}-08$ & & \\
\hline Pathology $\mathrm{T}$ stages (T4 vs. T3 vs. T2 vs. T1 vs. T0) & 1.42 & 0.0007 & 1.19 & 0.157 \\
\hline Pathology N stages (N3 vs. N2 vs. N1 vs. N0) & 1.65 & 2.52E-07 & 1.17 & 0.15 \\
\hline Sex (male vs. female) & 1.12 & 0.498 & & \\
\hline Ethnicity (white vs. non-white) & 1.74 & 0.211 & & \\
\hline miRNA model scores (high vs. low score) & 2.87 & 0.0002 & 1.62 & 0.005 \\
\hline
\end{tabular}

microRNA, miRNA.

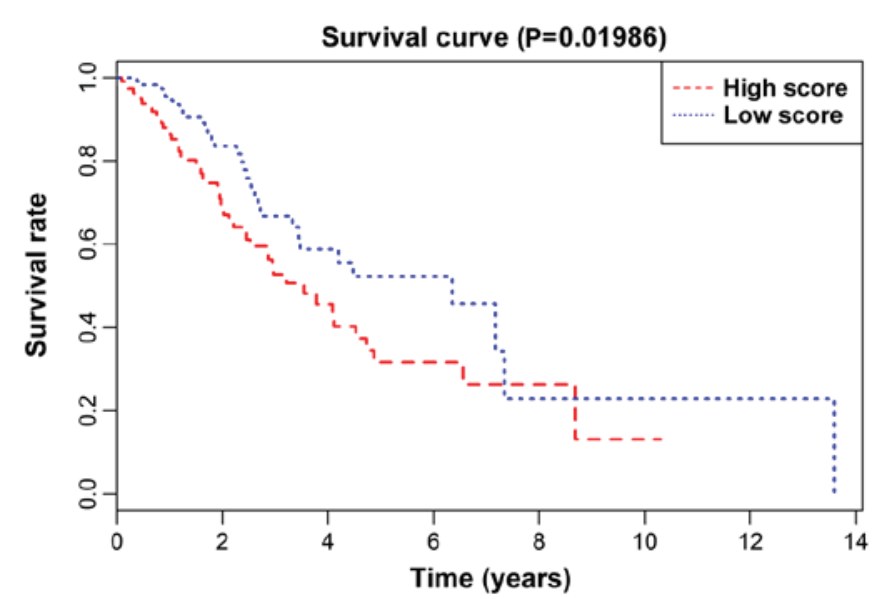

Figure 2. Kaplan-Meier survival curve of prognostic model in the training set.

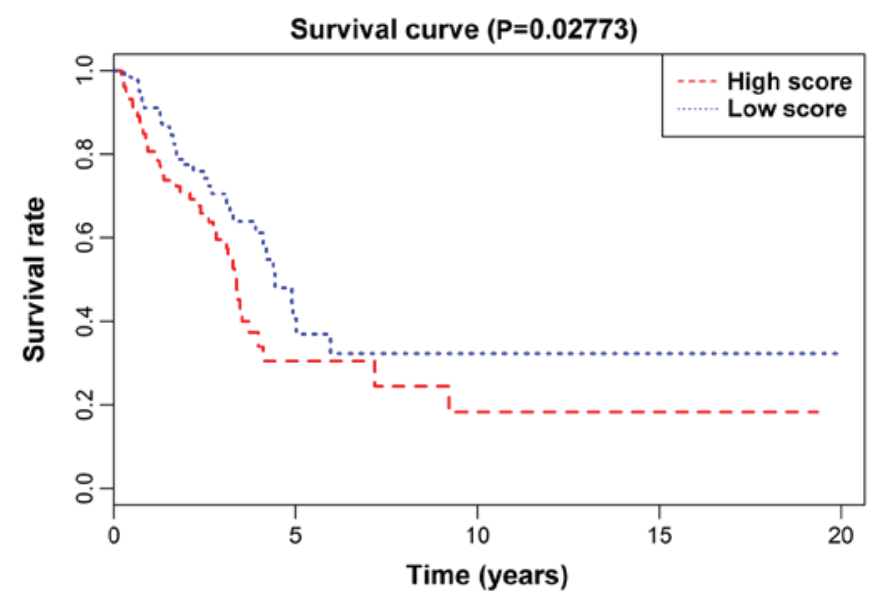

Figure 3. Kaplan-Meier survival curve of the prognostic model in the validation set.

In addition, the analysis through the single factor Cox regression showed that pathologic stages $(\mathrm{P}=2.22 \mathrm{E}-08)$, pathology $\mathrm{N}$ stages $(\mathrm{P}=2.52 \mathrm{E}-07)$, and pathology $\mathrm{T}$ stages $(\mathrm{P}=0.0007)$ were closely related to the survival of patients with lung adenocarcinoma (Table II). Multivariable Cox

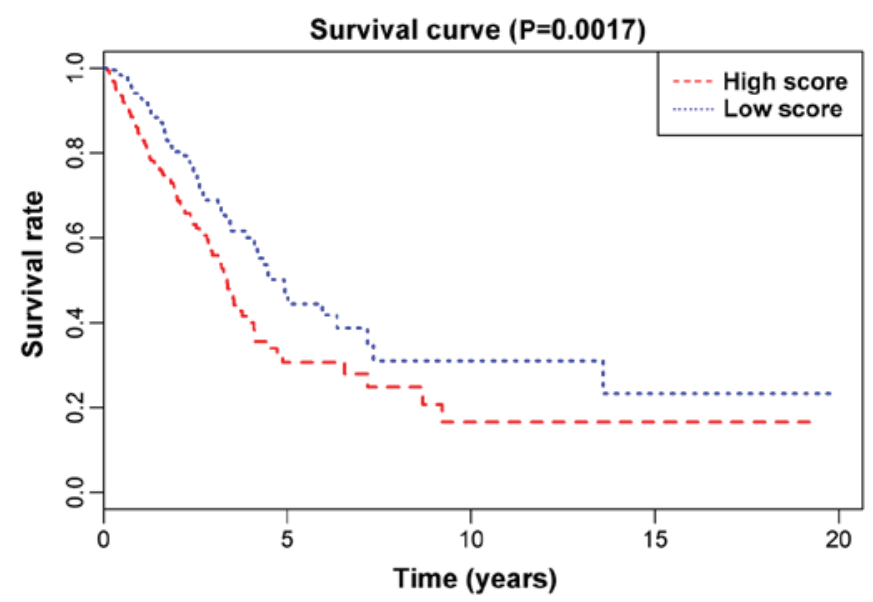

Figure 4. Kaplan-Meier survival curve of the prognostic model in the total number of patients.

regression showed both the prognosis model $(\mathrm{P}=0.005)$ and age $(\mathrm{P}=0.03)$ were independent prognostic variable models of lung adenocarcinoma (Table II). However, the prognosis model constructed in the study was superior to the assessment of the survival of patients with lung adenocarcinoma with age.

\section{Discussion}

By constructing a prognosis model focusing on four miRNAs, patients with lung adenocarcinoma were divided into a high- or low-risk group, and the survival time of the highrisk group was shown to be less than that of low-risk group. After constructing a multivariable Cox regression model using patient clinical characteristics, the results showed that the prognosis model serves as a potential independent prognostic model to assess the survival time of patients with lung adenocarcinoma $(\mathrm{P}=0.005)$. The prognostic model had a general assessment effect on the treatment $(\mathrm{P}=0.65091)$ and non-Caucasian $(\mathrm{P}=0.60825)$ groups, but were underpowered due to too few patients in the two groups. The prognostic model showed a good assessment effect on the non-treatment and Caucasian groups, and it could also predict the 5-year survival rate of patients with lung adenocarcinoma of the 
Caucasian group (AUC=0.629). As the majority of the data in TCGA included Caucasian patients, and contained data from only a relatively smaller proportion from other ethnicities, the conclusions drawn from the present study are more applicable to Caucasian patients.

The prognostic model from the present study utilized the miRNAs hsa-miR-101-1, hsa-miR-200a, hsa-miR-4661 and hsa-miR-450a-2. Chang et al (18) showed that hsa-miR-200a was downregulated in patients with gastric cancer, and could be used as a potential biomarker to predict the survival and prognosis of patients with gastric cancer. In the present study, there was an upregulated expression of hsa-miR-200a miRNA in lung adenocarcinoma tissue samples, indicating a differential regulation of the same miRNA from these different tumors. Liu et al (19) demonstrated that the expression of miR-101 in breast cancer could lead to E-cadherin downregulation, and miR-101 may inhibit the expression of DNMT3A and the proliferation and migration of human breast adenocarcinoma MDA-MB-231 cells. Furthermore, hsa-miR-101 is involved in a wide variety of other tumor processes [e.g., pancreatic cancer (20) and hepatocellular carcinoma (21)]. Other findings showed that the downregulated expression of miRNA-450b-3p could lead to the upregulated expression of HER3, thereby affecting the prognosis of patients with breast cancer (22). The prognosis model focusing on the four miRNAs constructed in the present study confirmed that the expression of these tissue miRNAs correlated with the survival time of patients with lung adenocarcinoma, and it can be used as an independent prognostic model for prognosis assessment of patients with lung adenocarcinoma.

Although the independent prognosis model for evaluating the patients with lung adenocarcinoma was established in the study, there are limitations. Firstly, all the data in the study were from one database, the TCGA, and the conclusions could be more reliable if they were verified using other independent databases. In addition, the study only included 478 patients with lung adenocarcinoma, and some of the subgroups were underpowered. In conclusion, the prognostic model developed in the present study, focusing on miRNAs, can be used as an independent prognostic model for survival time of patients with lung adenocarcinoma.

\section{References}

1. Jemal A, Siegel R, Xu J and Ward E: Cancer statistics, 2010. CA Cancer J Clin 60: 277-300, 2010.

2. Jemal A, Bray F, Center MM, Ferlay J, Ward E and Forman D: Global cancer statistics. CA Cancer J Clin 61: 69-90, 2011.

3. Siegel R, Naishadham D and Jemal A: Cancer statistics, 2013. CA Cancer J Clin 63: 11-30, 2013.
4. Herbst RS, Heymach JV and Lippman SM: Lung cancer. N Engl J Med 359: 1367-1380, 2008.

5. Imielinski M, Berger AH, Hammerman PS, Hernandez B, Pugh TJ, Hodis E, Cho J, Suh J, Capelletti M, Sivachenko A, et al: Mapping the hallmarks of lung adenocarcinoma with massively parallel sequencing. Cell 150: 1107-1120, 2012.

6. Peng F, Li H, Ning Z, Yang Z, Li H, Wang Y, Chen F and Wu Y: CD147 and prostate cancer: a systematic review and meta-analysis. PLoS One 11: e016367, 2016.

7. Toh CK, Gao F, Lim WT, Leong SS, Fong KW, Yap SP, Hsu AA, Eng P, Koong HN, Thirugnanam A and Tan EH: Never-smokers with lung cancer: epidemiologic evidence of a distinct disease entity. J Clin Oncol 24: 2245-2251, 2006.

8. Subramanian J and Govindan R: Lung cancer in never smokers: a review. J Clin Oncol 25: 561-570, 2007.

9. Gavelli G and Giampalma E: Sensitivity and specificity of chest X-ray screening for lung cancer: review article. Cancer 89 (Suppl): 2453-2456, 2000.

10. Hayes J, Peruzzi PP and Lawler S: MicroRNAs in cancer: biomarkers, functions and therapy. Trends Mol Med 20: 460-469, 2014.

11. Zhao Z, Li S, Song E and Liu S: The roles of ncRNAs and histone-modifiers in regulating breast cancer stem cells. Protein Cell 7: 89-99, 2016.

12. O'Kelly F, Marignol L, Meunier A, Lynch TH, Perry AS and Hollywood D: MicroRNAs as putative mediators of treatment response in prostate cancer. Nat Rev Urol 9: 397-407, 2012.

13. Zhou L, Zhao LC, Jiang N, Wang XL, Zhou XN, Luo XL and Ren J: MicroRNA miR-590-5p inhibits breast cancer cell stemness and metastasis by targeting SOX2. Eur Rev Med Pharmacol Sci 21: 87-94, 2017.

14. Feng Y, Liu J, Kang Y, He Y, Liang B, Yang P and Yu Z: miR-19a acts as an oncogenic microRNA and is up-regulated in bladder cancer. J Exp Clin Cancer Res 33: 67, 2014.

15. Robinson MD, McCarthy DJ and Smyth GK: edgeR: a Bioconductor package for differential expression analysis of digital gene expression data. Bioinformatics 26: 139-140, 2010.

16. Posada D and Buckley TR: Model selection and model averaging in phylogenetics: advantages of Akaike information criterion and Bayesian approaches over likelihood ratio tests. Syst Biol 53: 793-808, 2004.

17. Huang W, Sherman BT and Lempicki RA: Systematic and integrative analysis of large gene lists using DAVID bioinformatics resources. Nat Protoc 4: 44-57, 2009.

18. Chang L, Guo F, Huo B, Lv Y, Wang Y and Liu W: Expression and clinical significance of the microRNA-200 family in gastric cancer. Oncol Lett 9: 2317-2324, 2015.

19. Liu J, Pang Y, Wang H, Li Y, Sun X, Xu F, Ren H and Liu D: miR-101 inhibits the proliferation and migration of breast cancer cells via downregulating the expression of DNA methyltransferase 3a. Xi Bao Yu Fen Zi Mian Yi Xue Za Zhi 32: 299-303, 2016 (In Chinese).

20. Fan P, Liu L, Yin Y, Zhao Z, Zhang Y, Amponsah PS, Xiao X, Bauer N, Abukiwan A, Nwaeburu CC, et al: MicroRNA-101-3p reverses gemcitabine resistance by inhibition of ribonucleotide reductase M1 in pancreatic cancer. Cancer Lett 373: 130-137, 2016.

21. Cao K, Li J, Zhao Y, Wang Q, Zeng Q, He S, Yu L, Zhou J and Cao P: miR-101 inhibiting cell proliferation, migration and invasion in hepatocellular carcinoma through downregulating Girdin. Mol Cell 39: 96-102, 2016.

22. Zhao Z, Li R, Sha S, Wang Q, Mao W and Liu T: Targeting HER3 with miR-450b-3p suppresses breast cancer cells proliferation. Cancer Biol Ther 15: 1404-1412, 2014. 